\title{
THE ISOMORPHISM THEOREM IN COMPACTLY GENERATED LATTICES
}

\author{
BY PETER CRAWLEY
}

\author{
Communicated by R. S. Pierce, October 5, 1959
}

It is a well known property of a modular lattice $L$ that if $a, b \in L$ then the quotient sublattices $a \cup b / a$ and $b / a \cap b$ are isomorphic. Morgan Ward [3] has proved that if the ascending or descending chain condition holds, then this property is equivalent to modularity. If the chain conditions are dropped, however, then there are simple examples of nonmodular lattices $L$ for which $a \cup b / a$ and $b / a \cap b$ are isomorphic for all $a, b \in L$.

We shall show here that the isomorphism of all transposed quotients does in general characterize modularity, provided this condition is applied to the ideals of the lattice. More precisely, this result is the following. If $L$ is an arbitrary lattice, then $L$ is modular if and only if for every pair of ideals $A, B$ of $L$, the quotients of ideals $A \cup B / A$ and $B / A \cap B$ are isomorphic.

Actually this result is a corollary of a more general theorem on compactly generated lattices. An element $c$ in a complete lattice $L$ is compact if for every subset $S \subseteq L$ with $c \leqq \bigcup S$ there exists a finite subset $S^{\prime} \subseteq S$ such that $c \leqq \mathrm{U} S^{\prime}$. A lattice $L$ is said to be compactly generated if $L$ is complete and every element of $L$ is a join of compact elements.

Theorem. Let $L$ be a compactly generated lattice. If $a \cup b / a$ and $b / a \cap b$ are isomorphic for all $a, b \in L$, then $L$ is modular.

As the lattice of congruence relations of any algebraic system is compactly generated, compactly generated lattices play a fundamental role in the decomposition theory of algebraic systems, and in this regard the theorem would seem a natural one. ${ }^{1}$ It is clear that for any lattice $L$, the lattice $I(L)$ of all ideals of $L$ is compactly generated. Thus, since $I(L)$ is modular if and only if $L$ is modular, the italicized statement in the second paragraph is a direct consequence of the theorem.

Throughout this note the usual notation and terminology is used. Lattice inclusion, proper inclusion, and covering, are denoted by the symbols $\leqq,<,<$, respectively. The quotient sublattice $a / b$ is defined by $a / b=\{x \in L \mid b \leqq x \leqq a\}$.

${ }^{1}$ For the decomposition theory associated with compactly generated lattices see Dilworth-Crawley [2]. 
The proof of the theorem requires the following three lemmas.

Lemma 1. Let $L$ be a compact generated lattice. If $a, b \in L$ and $a>b$, then there exist elements $p, q \in L$ such that $a \geqq p>q \geqq b$.

Lemma 2. Let $L$ be a compactly generated lattice. If $\left\{x_{\alpha}\right\}$ is a chain of elements of $L$ and $a \in L$, then $a \cap \bigcup_{\alpha} x_{\alpha}=\cup_{\alpha} a \cap x_{\alpha}$.

Lemma 3. Let $L$ be a lattice such that $a \cup b / a \cong b / a \cap b$ for all $a, b \in L$. Then if $p, q, r \in L$ and $p>q$, either $r \cap p=r \cap q$ or $r \cap p>r \cap q$. The dual statement also holds.

Lemma 1 is given in [1, Lemma 2.4], Lemma 2 is essentially given in [2, Lemma 2.3], and Lemma 3 is given in [3, Lemmas 5.6].

We may now proceed with the proof of the theorem. Suppose $L$ is a compactly generated lattice satisfying the hypothesis of the theorem, but $L$ is not modular. Then $L$ contains a five-element sublattice $\{a, b, t, u, v\}$ such that $a>b$ and $t \cup a=t \cup b=u, t \cap a=t \cap b=v$. If $a$ does not cover $b$, then by Lemma 1 there exist elements $p, q \in L$ such that $a \geqq p>q \geqq b$. Clearly $t \cup p=t \cup q=u$ and $t \cap p=t \cap q=v$. Thus the sublattice $\{p, q, t, u, v\}$ is a nonmodular five-element sublattice in which $p>q$. Hence we may assume that the sublattice $\{a, b, t, u, v\}$ was originally picked in such a way that $a>b$.

Let $T$ be the set of all ordered triples $(x, y, z), x, y, z \in L$, such that $x \geqq a, y \geqq b, z \geqq v$, and such that the following relations hold:

(i) $t \cup y=u$,

(ii) $t \cap x=z$,

(iii) $x>y$,

(iv) $a \cap y=b$.

$T$ is nonempty since the triple $(a, b, v)$ is in $T$. Now partially order $T$ by defining $(x, y, z) \leqq\left(x^{\prime}, y^{\prime}, z^{\prime}\right)$ if and only if $x \leqq x^{\prime}, y \leqq y^{\prime}$, and $z \leqq z^{\prime}$. Suppose $\left\{\left(x_{\alpha}, y_{\alpha}, z_{\alpha}\right)\right\}$ is a chain of $T$. Let $\bar{x}=U_{\alpha} x_{\alpha}, \bar{y}=U_{\alpha} y_{\alpha}$, and $\bar{z}=\cup_{\alpha} z_{\alpha}$. By infinite associativity and Lemma 2 we have, $t \cup_{\bar{y}}$ $=t \cup_{\alpha} y_{\alpha}=\cup_{\alpha} t \cup y_{\alpha}=u, \quad t \cap \bar{x}=t \cap \bigcup_{\alpha} x_{\alpha}=\bigcup_{\alpha} t \cap x_{\alpha}=\bigcup_{\alpha} z_{\alpha}=\bar{z}$, and $a \cap \bar{y}=a \cap \bigcup_{\alpha} y_{\alpha}=\cup_{\alpha} a \cap y_{\alpha}=b$. Note that for each index $\alpha, a \cup y_{\alpha}=x_{\alpha}$ since $x_{\alpha} \geqq a$ and $x_{\alpha}>y_{\alpha}$. Thus $a \cup_{\bar{y}}=a \cup \cup_{\alpha} y_{\alpha}=\bigcup_{\alpha} a \cup y_{\alpha}=\bigcup_{\alpha} x_{\alpha}=\bar{x}$. Hence $\bar{x} / \bar{y}=a \cup_{\bar{y}} / \bar{y} \cong a / a \cap \bar{y}=a / b$ by hypothesis, and since $a>b$ we must have $\bar{x}>\bar{y}$. Thus the triple $(\bar{x}, \bar{y}, \bar{z}) \in T$, and every chain of $T$ has an upper bound. By the Maximal Principle, $T$ contains a maximal element $\left(a_{0}, b_{0}, v_{0}\right)$.

Now $u / b_{0}=t \cup b_{0} / b_{0} \cong t / t \cap b_{0}=t / v_{0}$, and since $u>a_{0}>b_{0}$, there must exist an element $v_{1} \in L$ such that $t>v_{1}>v_{0}$. Let $a_{1}=a_{0} \cup v_{1}$ and $b_{1}$ 
$=b_{0} \cup v_{1}$. We shall show that the triple $\left(a_{1}, b_{1}, v_{1}\right)$ satisfies conditions (i)-(iv).

It is clear that $t \cup b_{1}=u$. Notice that $a_{0} \geq v_{1}$, since $t \cap a_{0}=v_{0}$. Thus $a_{1}=a_{0} \cup v_{1} \neq a_{0} \cup v_{0}=a_{0}$, and hence by Lemma $3, a_{1}>a_{0}$ since $v_{1}>v_{0}$. Computing further, $t \cap a_{1} \geqq v_{1}>v_{0}=t \cap a_{0}$, and hence by Lemma 3 , $t \cap a_{1}>v_{0}$. Thus $t \cap a_{1}=v_{1}$. Just as $a_{1}>a_{0}$, it follows that $b_{1}>b_{0}$. Hence $a_{1} \neq b_{1}$, for otherwise $b_{1}>a_{0}>b_{0}$, contrary to $b_{1}>b_{0}$. Thus by Lemma 3 and the fact that $a_{0}>b_{0}$, we must have $a_{1}>b_{1}$. Finally, consider $a \cap b_{1}$. Since $a_{0} \geqq a>b$ and $b_{1}>b$, it follows that $a \cap b_{1} \leqq a_{0} \cap b_{1}=b_{0}$. Hence $b \leqq a \cap b_{1} \leqq a \cap b_{0}=b$; whence $a \cap b_{1}=b$. Thus the triple $\left(a_{1}, b_{1}, v_{1}\right)$ satisfies conditions (i) -(iv), and hence $\left(a_{1}, b_{1}, v_{1}\right) \in T$. But $\left(a_{1}, b_{1}, v_{1}\right)$ is properly bigger than $\left(a_{0}, b_{0}, v_{0}\right)$, and since $\left(a_{0}, b_{0}, v_{0}\right)$ was picked to be a maximal element of $T$, we have a contradiction. Thus $L$ must be modular, and the proof of the theorem is complete.

\section{REFERENCES}

1. P. Crawley, Lattices whose congruences form a Boolean algebra, Pacific J. Math. (to appear.)

2. R. P. Dilworth and P. Crawley, Decomposition theory for lattices without chain conditions, to appear in Trans. Amer. Math. Soc.

3. Morgan Ward, $A$ characterization of Dedekind structures, Bull. Amer. Math. Soc. vol. 45 (1939) pp. 448-451.

California Institute of Technology 